\title{
Zinc finger E-box-binding homeobox 1 : its clinical significance and functional role in human thyroid
} \section{cancer}

This article was published in the following Dove Press journal:

OncoTargets and Therapy

29 March 2016

Number of times this article has been viewed

Yan Zhang*

Gang Liu*

Shihe Wu

Futing Jiang

Jiangping Xie

Yuhong Wang

Department of General Surgery, Navy General Hospital of Chinese PLA, Beijing, People's Republic of China

*These authors contributed equally to this work
Correspondence: Yuhong Wang Department of General Surgery, Navy General Hospital of Chinese PLA, No 28, Fuxing street, Haidian District, Beijing 100048, People's Republic of China Tel +861067355289 Fax +86 I0 67355289 Email yhwanghospital@I63.com
Objective: Transcription factor zinc finger E-box-binding homeobox 1 (ZEB1), as one of the key inducers of epithelial-mesenchymal transition, has been reported to be regulated by microRNA144 and Bcl-2-associated athanogene 3, which both promote thyroid cancer cell invasion. However, the involvement of ZEB1 in thyroid cancer has not been fully elucidated. In this study, we aimed to investigate the role and clinical implication of ZEB1 in this disease.

Methods: Immunohistochemistry was performed to examine the subcellular localization and the expression level of ZEB1 protein in 82 self-pairs of formalin-fixed and paraffin-embedded cancerous and adjacent noncancerous tissues obtained from patients with thyroid cancer. The roles of ZEB1 in thyroid cancer cell migration, invasion, and proliferation were also detected by transwell and MTT analyses, respectively.

Results: Immunohistochemistry showed that ZEB1 was predominantly localized in the nucleus of thyroid cancer cells. Its immunoreactive score in thyroid cancer tissues was significantly higher than that in adjacent noncancerous tissues $(P=0.01)$. In addition, $Z E B 1$ overexpression was significantly associated with the advanced tumor node metastasis staging $(P=0.008)$, the positive lymph node metastasis $(P=0.01)$ and distant metastasis $(P=0.02)$. Furthermore, ZEB1 knockdown by siRNA could efficiently inhibit the migration, invasion, and proliferation abilities of thyroid cancer cells in vitro.

Conclusion: These findings indicated that ZEB1 might function as an oncogene, the overexpression of which was associated with the aggressive tumor progression of human thyroid cancer. Interestingly, ZEB1 also could promote thyroid cancer cell migration, invasion, and proliferation, suggesting that the inhibition of this protein might be a therapeutic strategy for the treatment of this malignancy.

Keywords: zinc finger E-box-binding homeobox 1, thyroid cancer, migration, invasion, proliferation, immunohistochemistry, small interfering RNA

\section{Introduction}

Thyroid cancers, originating from follicular epithelial cells, represent a group of the most common endocrine cancers and consist of various malignant phenotypes ranging from benign adenomas and well-differentiated carcinoma to poorly differentiated thyroid carcinoma and the rare but rapidly lethal anaplastic (or undifferentiated) thyroid carcinoma. ${ }^{1}$ Among them, well-differentiated carcinomas are classified into two types: papillary thyroid cancer and follicular thyroid cancer, which both have a favorable prognosis, but the overall recurrence rates may be $\sim 35 \% ;^{2}$ anaplastic thyroid carcinoma is often recognized as a rapidly increasing neck mass that spreads locally, compresses the adjacent structures, with a tendency to disseminate to regional lymph nodes and distant sites. ${ }^{3}$ In recent years, surgical intervention and radioiodine therapy have been extensively used 
for the treatment of patients with thyroid cancers, however, a number of patients have developed disease recurrence, aggressiveness, or metastasis. ${ }^{4,5}$ Although accumulating studies have revealed the molecular mechanisms underlying the carcinogenesis and cancer progression of this malignancy, there is a lack of molecular markers to predict the aggressiveness of thyroid cancer. Thus, it is of great significance to clarify the critical molecular steps of thyroid cancer progression in order to identify efficient diagnostic and prognostic markers for patients with this disease.

It is a multistage process from carcinogenesis to metastatic malignancy, in which cancer cells gain the ability to invade and colonize distant tissues. During this process, epithelialmesenchymal transition (EMT) is implicated into the invasive conversion of epithelial cells. ${ }^{6}$ The zinc finger E-box-binding homeobox 1 (ZEB1) transcription factor (also called AREB6, BZP, Nil-2-a, TCF8, dEF1, zfhx1a, and Zfhep in vertebrates) has been recognized as a critical inducer of EMT in some cell types and reported to be involved in maintaining the epithelial phenotype and activating those required for transformation to the mesenchymal phenotype. ${ }^{7,8}$ As a nuclear factor, ZEB1 has been observed to be abnormally expressed by various types of cancer cells and play a crucial roles during cancer progression. ${ }^{9}$ Especially, Guan et al ${ }^{10}$ verified ZEB1 as a direct target of microRNA-144, which was significantly downregulated in thyroid cancer as compared with that in normal thyroid tissues, and the restoration of which in thyroid cancer cells could suppress the invasion and migration capability of these cells; Meng et $\mathrm{al}^{11}$ indicated that ZEB1 expression was regulated by Bcl-2-associated athanogene 3, which might function as a regulator of metastasis in thyroid cancer cells. However, the involvement of ZEB1 in thyroid cancer has not been fully elucidated. Therefore, we in the current study aimed to investigate the role and clinical implication of ZEB1 in this disease.

\section{Materials and methods}

\section{Ethics statement}

All participants gave written informed consent to participate in this study, which was approved by the Research Ethics Committee of Navy General Hospital of Chinese PLA, Beijing, People's Republic of China. This study was conducted according to the principles of the Declaration of Helsinki. All specimens were handled and made anonymous based on the ethical and legal standards.

\section{Patients and tissue samples}

Eighty-two self-pairs formalin-fixed and paraffin-embedded cancerous and adjacent noncancerous tissues were obtained from thyroid cancer patients who underwent total thyroidectomy at Department of General Surgery, Navy General Hospital of Chinese PLA between March 2008 and April 2014. The diagnoses of thyroid cancers were derived from histopathological analyses and clinical examination. There were 22 men and 60 women ranging in age from 32 to 82 years (median age 50 years). Tumor stage was performed based on the sixth edition of the tumor-node metastasis (TNM) classification of the International Union Against Cancer. The patient characteristics, including patients' age, sex, tumor size, histological type, TNM stage, the presence of lymph nodes and distant metastases, are summarized in Table 1.

\section{Cell culture}

Two human thyroid cancer cell lines ARO and TT were purchased from the Cell Bank of the Chinese Academy of Medical Sciences (Beijing, People's Republic of China). All cells were cultured in Dulbecco's Modified Eagle's Medium (DMEM) (Thermo Fisher Scientific, Waltham, MA, USA) supplemented with $10 \%$ fetal calf serum (Thermo Fisher Scientific) in a humidified atmosphere of $5 \% \mathrm{CO}_{2}$ at $37^{\circ} \mathrm{C}$.

\section{Immunohistochemistry}

Serial thyroid cancer tissue sections ( $4 \mu \mathrm{m}$ thickness) were deparaffinized, hydrated in xylene, and graded alcohol series. The endogenous peroxidase activity was inactivated in a solution containing 3\% hydrogen peroxide in methanol. After blocking the nonspecific binding with normal goat serum, the sections were incubated with the rabbit anti-ZEB1 (1:500 dilution; \# sc-25388, Santa Cruz Biotechnology, Santa Cruz, CA, USA) at $4^{\circ} \mathrm{C}$ overnight, and then with the biotinylated secondary antibody after being washed with Tris-buffered $\mathrm{NaCl}$ solution for 30 minutes. After that, the sections were incubated with VECTASTAIN Elite ABC Reagent (Vector Laboratories, Burlington, ON, Canada) according to the manufacturer's instructions and diaminobenzidine was used as the chromogen. No immunoreactivity was observed in tissue sections where the primary antibody was replaced by isotype-matched irrelevant antibody and was used as a negative control. The thyroid cancer tissues with the overexpression of ZEB1 protein were confirmed by Western blot and was used as a positive control.

To evaluate the immunostaining of ZEB1 protein, all sections were scored by two independent pathologists who were blinded to patients' characteristics, and any discrepancies were resolved by consensus. Sections were scored as positive 
Table I Associations between ZEBI expression and various clinicopathological characteristics of patients with thyroid cancer

\begin{tabular}{|c|c|c|c|c|}
\hline \multirow[t]{2}{*}{ Clinicopathological features } & \multirow[t]{2}{*}{ Number of cases } & \multicolumn{2}{|c|}{ ZEB I expression } & \multirow[t]{2}{*}{$P$-value } \\
\hline & & High n, (\%) & Low $n,(\%)$ & \\
\hline \multicolumn{5}{|l|}{ Age (years) } \\
\hline$<50$ & 32 & $18(56.25)$ & $14(43.75)$ & NS \\
\hline$\geq 50$ & 50 & $24(48.00)$ & $26(52.00)$ & \\
\hline \multicolumn{5}{|l|}{ Sex } \\
\hline Male & 22 & $12(54.55)$ & $10(45.45)$ & NS \\
\hline Female & 60 & $30(50.00)$ & $30(50.00)$ & \\
\hline \multicolumn{5}{|l|}{ Tumor size $(\mathrm{cm})$} \\
\hline$\leq 5$ & 48 & $25(52.08)$ & $23(47.92)$ & NS \\
\hline$>5$ & 34 & $17(50.00)$ & $17(50.00)$ & \\
\hline \multicolumn{5}{|l|}{ Histological type } \\
\hline Follicular carcinoma & 38 & $20(52.63)$ & $18(47.37)$ & NS \\
\hline Papillary carcinoma-classic variant & 32 & $16(50.00)$ & $16(50.00)$ & \\
\hline Papillary carcinoma-tall cell variant & 12 & $6(50.00)$ & $6(50.00)$ & \\
\hline \multicolumn{5}{|l|}{ TNM stage } \\
\hline I-II & 53 & $17(32.08)$ & $36(67.92)$ & 0.008 \\
\hline III-IV & 29 & $25(86.21)$ & $4(13.79)$ & \\
\hline \multicolumn{5}{|l|}{ Lymph nodes metastasis } \\
\hline Negative & 50 & $16(32.00)$ & $34(68.00)$ & 0.01 \\
\hline Positive & 32 & $26(8 I .25)$ & $6(18.75)$ & \\
\hline \multicolumn{5}{|l|}{ Distant metastasis } \\
\hline Negative & 55 & $22(40.00)$ & $33(60.00)$ & 0.02 \\
\hline Positive & 27 & $20(74.07)$ & $7(25.93)$ & \\
\hline
\end{tabular}

Note: "NS" refers to the difference without statistical significance.

Abbreviations: TNM, tumor-node metastasis; ZEBI, zinc finger E-box-binding homeobox I.

if epithelial cells showed immunopositivity in nucleus.

These sections were scored using a semi-quantitative scoring system combining the staining intensity with the percentage of positive cells as described by the previous studies. ${ }^{12,13}$ The intensity was classified as follows: 0 , negative staining; 1 , weak staining; 2 , moderate staining; 3 , strong staining. The percent of positive cells was judged followed by Remmele W's criteria 17 : 1) $0 \%-25 \%$; 2) $26 \%-50 \%$; 3) $51 \%-75 \%$; 4) $>75 \%$. A final immunoreactive score (IRS) was achieved by multiplying the intensity and the percent of positive cells. To divide all 82 patients with thyroid cancer into high ZEB1 (ZEB1-high) and low ZEB1 (ZEB1-low) expression groups, the median value of ZEB1 protein IRS was used as a cutoff point.

\section{RNA interference of ZEBI}

ARO and TT cells in the logarithmic growth phase were transfected with non-targeting negative control small interfering RNA (siRNA, si-con; Santa Cruz Biotechnology) and human ZEB1 specific siRNA (si-ZEB1; Santa Cruz Biotechnology) using Lipofectamine 2000 (Thermo Fisher Scientific) according to the manufacturer's instructions. Cells were verified and used for analysis at 48 hours after transfection.

\section{Western blot analysis}

Expression levels of ZEB1 protein in ARO and TT cells were detected by Western blot analysis at 48 hours after transient transfection. In brief, $20 \mu \mathrm{g}$ of the total protein sample was separated by sodium dodecyl sulfate-polyacrylamide gel electrophoresis and transferred onto a polyvinylidene difluoride membrane (Amresco, Solon, OH, USA), which was blocked in TBST (10 mM Tris-HCl, $\mathrm{pH} 7.5,150 \mathrm{mM}$ $\mathrm{NaCl}$, and $0.1 \%$ Tween-20) with $5 \%$ skim milk at room temperature for 1 hour. Then, the rabbit anti-ZEB1 polyclonal antibody (1:150 dilution; \# sc-25388, Santa Cruz Biotechnology) and rabbit anti-GAPDH polyclonal antibody (1:1,000 dilution; Santa Cruz Biotechnology), which was used as an internal control for the normalization of candidate proteins, were added and incubated overnight at $4^{\circ} \mathrm{C}$. Following that, the horseradish peroxidase-labeled goat anti-rabbit IgG polyclonal antibody (1:2,000 dilution; Santa Cruz Biotechnology) was added and the membrane was incubated at $37^{\circ} \mathrm{C}$ for 1 hour. Protein expression was visualized by enhanced chemiluminescence and exposure to chemiluminescent film (Bucks, Amersham, UK). Band intensities were quantified by densitometry. The relative expression of ZEB1 was represented as the band intensity ratio of ZEB1/GAPDH. 


\section{Cell migration and invasion assays}

The abilities of ARO and TT cells to migrate and invade were both evaluated by using transwell inserts (24-well insert, pore size $8 \mu \mathrm{m}$; EMD Millipore, Billerica, MA, USA). ARO and TT cells transfected with si-ZEB1 and non-targeting negative si-con were treated with trypsin/EDTA solution, washed once with serum-containing DMEM medium, and seeded in the upper chamber at a density of $2 \times 10^{5}$ cells $/ \mathrm{mL}$ in $400 \mu \mathrm{L}$ of medium containing $10 \%$ fetal bovine serum (FBS). DMEM medium containing $10 \%$ FBS was added into the lower chamber as a chemoattractant. The cell invasion assay was conducted following the same procedure as mentioned above, with the exception that the filters of the transwell chambers were coated with $45 \mu \mathrm{g}$ Matrigel (BD Biosciences, San Jose, CA, USA). Following a 24 hours incubation at $37^{\circ} \mathrm{C}$ with $5 \% \mathrm{CO}_{2}$, nonmigrating/noninvading cells in the upper chamber were removed with a cotton swab, and migrating/ invading cells were fixed in $100 \%$ methanol and stained with $0.5 \%$ crystal violet in $2 \%$ ethanol. The number of migrating/ invading cells were manually counted at $200 \times$ magnification from ten different fields of each filter. All assays were performed in triplicate and the mean values were calculated.

\section{Cell proliferation assay}

Cell proliferation abilities of ARO and TT cells transfected with si-ZEB1 and non-targeting negative si-con were detected by the MTT assay according to the previous study. The absorbance at wavelength $570 \mathrm{~nm}$ was measured for the supernatant of each well using the ELISA plate reader (Model 550; BioRad Laboratories Inc., Hercules, CA, USA). All assays were performed in triplicate and the mean values were calculated.

\section{Statistical analysis}

The results were expressed as mean $\pm \mathrm{SD}$. The statistical significance between two groups was evaluated via a $t$-test or one-way analysis of variance using SPSS 11.0 software (SPSS Inc., Chicago, IL, USA). The associations between ZEB1 expression and various clinicopathological features were calculated using the Fisher's exact test and chi-square. $P<0.05$ was considered to be a statistically significant result.

\section{Results}

\section{Overexpression of ZEBI protein in human thyroid cancer tissues}

Immunohistochemistry showed that ZEB1 was predominantly localized in the nucleus of thyroid cancer cells (Figure 1A), while faint or negative staining was observed in adjacent noncancerous tissues (Figure 1B). Positive immunostaining of ZEB1 protein was observed in $60(73.17 \%)$ of 82 patients with thyroid cancer, and its IRS in thyroid cancer tissues was significantly higher than that in adjacent noncancerous tissues $(P=0.01$, Figure $1 C)$.

Based on our immunochemistry scoring system, an optimal cutoff value (4.37) was identified: the high ZEB1 expression group ZEB1-high, ZEB1 expression level was higher than the cutoff value; median expression value 5.88, $\mathrm{n}=42,51.22 \%$ and the low ZEB1 expression group ZEB1low, ZEB1 expression level was lower than the cutoff value; median expression value $3.33, \mathrm{n}=40,48.78 \%$.

\section{Overexpression of ZEBI protein associates with aggressive progression of patients with thyroid cancer}

Table 1 summarized the associations of ZEB1 protein expression with various clinicopathological characteristics of patients with thyroid cancer. The expression levels of ZEB1 protein in patients with advanced TNM stage were more frequently higher than those with early TNM stage $(P=0.008)$. In addition, high ZEB1 protein expression was positively correlated with the presence of lymph node metastasis $(P=0.01)$ and distant
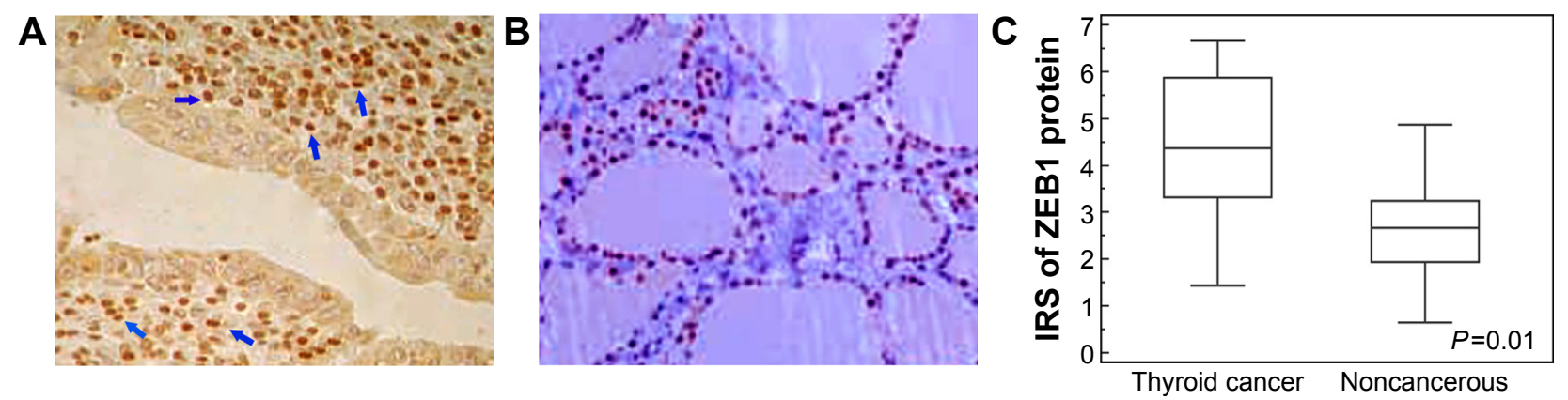

Figure I Immunostaining of ZEBI protein in human thyroid cancer $(\mathbf{A})$ and adjacent noncancerous tissues (B). (C) Expression level of ZEBI protein in thyroid cancer tissues was significantly higher than in adjacent noncancerous tissues $(P=0.0 \mathrm{I})$.

Note: The blue arrows indicate the positive staining of ZEBI protein in glioma tissues.

Abbreviations: IRS, immunoreactive score; ZEBI, zinc finger E-box-binding homeobox I. 


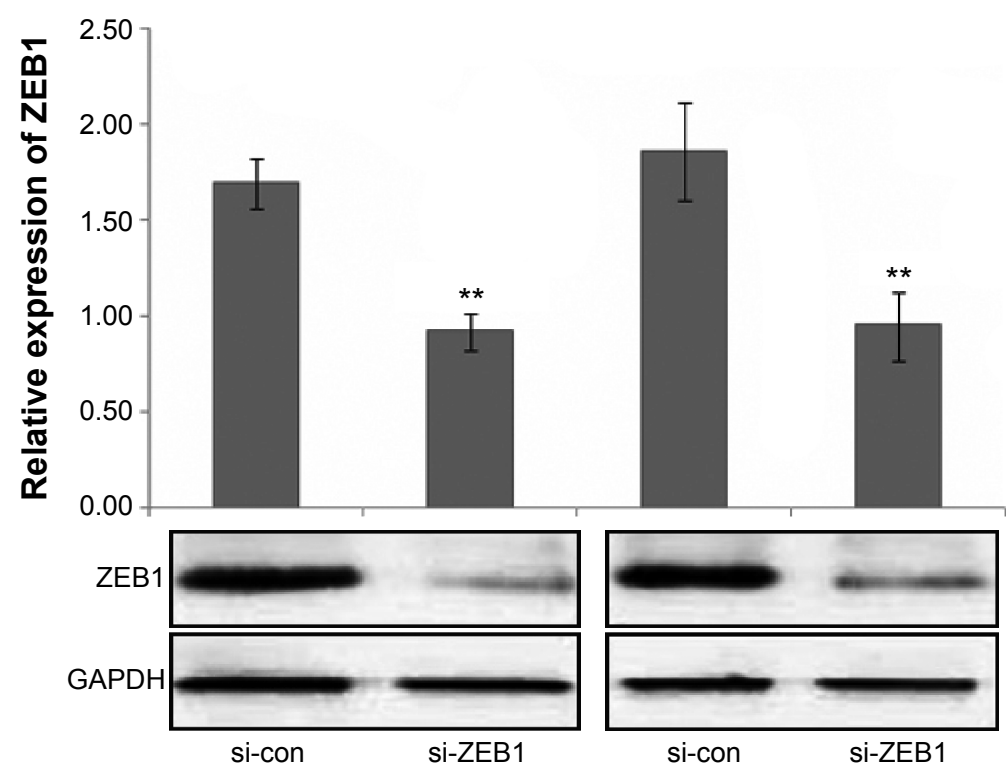

Figure 2 Relative expression of ZEBI protein in ARO and TT cells transfected with si-ZEBI and si-con.

Note: ${ }^{* *} P<0.01$ when compared with control cells.

Abbreviation: ZEBI, zinc finger E-box-binding homeobox I.

metastasis $(P=0.02)$. However, there were no significant associations between ZEB1 expression, and patients' age and sex, tumor size, and histological type (all $P>0.05$, Table 1).

\section{Knocking down ZEBI expression suppresses the migration, invasion, and proliferation potentials of thyroid cancer cell lines}

To determine the influence of ZEB1 on the migration, invasion, and proliferation of thyroid cancer cells, we transfected the ZEB1 RNAi plasmid into ARO and TT cells and performed transwell migration and invasion assays. Western blot analysis indicated that the transfection of RNAi plasmid that against ZEB1 could markedly inhibit the expression of endogenous ZEB1 protein in both ARO and TT cells (both $P<0.01$, Figure 2).

Interestingly, the migratory activities of si-ZEB1 transfected ARO and TT cells were both dramatically reduced, and the loss of ZEB1 expression in both cell lines also reduced the cell invasion potentials in the Matrigel substrate (all $P<0.01$, Figure 3). Moreover, we found that $42.06 \% \pm 1.22 \%$ and $46.51 \% \pm 0.98 \%$ cell proliferation inhibition 72 hours after siRNA transfection, respectively, in ARO and TT cells with stable knockdown of ZEB1, compared with that of the control (Figure 4).

\section{Discussion}

In the current report, we characterized the expression pattern of ZEB1 in human thyroid cancer tissues. Our results showed that ZEB1 expression was significantly increased in human thyroid cancer tissues, implying its involvement into carcinogenesis of this malignancy. The strong correlation between ZEB1 expression and thyroid cancer patients' TNM stage, the status of lymph node and distant metastasis highlighted the potential value of ZEB1 as a novel biomarker for human thyroid cancer progression. More importantly, we also described a previously unknown mechanism for the regulation of thyroid cancer cell migration and invasion. Our data found that ZEB1 could regulate the migration, invasion, and proliferation of thyroid cancer cells. These observations strongly suggest that ZEB1 may be acting as an oncogene to promote an aggressive tumor progression and a rapid metastasis of thyroid cancer cells.

ZEB1, a member of the ZEB family of transcription factors, is located on the short arm of human chromosome ten, with a coding sequence length of 3,327 bp encoding 1,108 amino acids, and contains two zinc finger clusters and a homeodomain. ${ }^{14}$ It can regulate the transcription of targeted genes by binding specific sequences of DNA using its zinc finger clusters. ${ }^{15}$ Growing evidence shows that ZEB1 plays an important role in cancer occurrence and development. For example, Liu et $\mathrm{al}^{16}$ reported that ZEB1 promoted the vasculogenic mimicry formation by inducing EMT in colorectal carcinoma and might represent an important target in this malignancy; Zhang et al ${ }^{17}$ also confirmed the marked correlations of ZEB1 overexpression with liver metastasis and poor prognosis in colorectal cancer; Kenney et al ${ }^{18}$ identified 


\section{A}

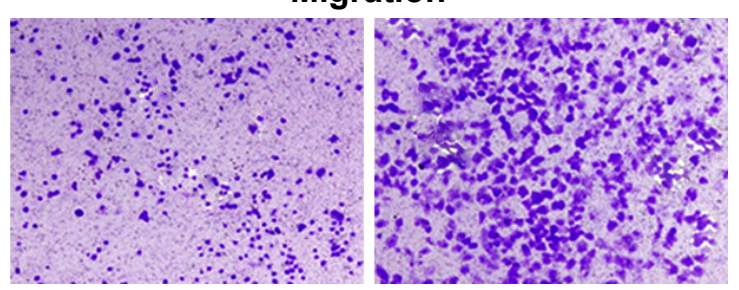

Invasion
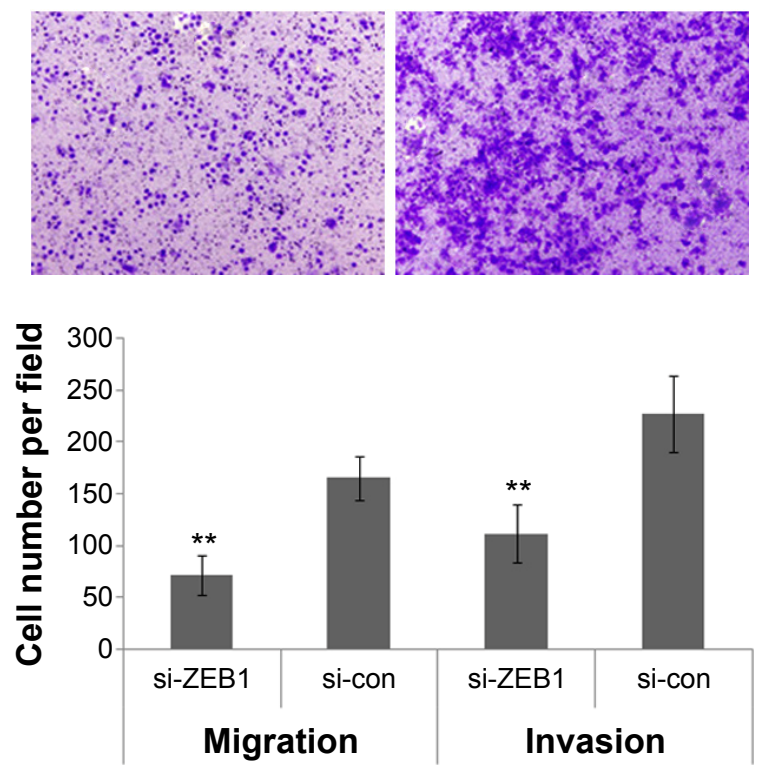

\section{B}

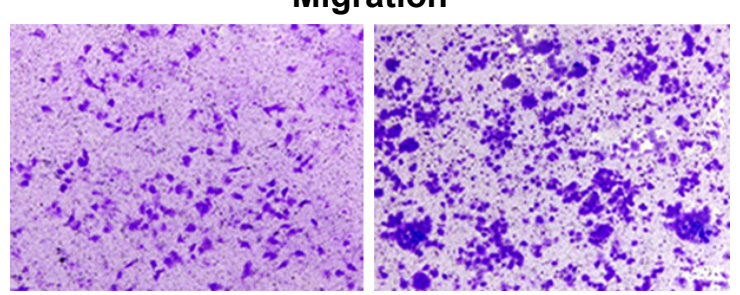

Invasion
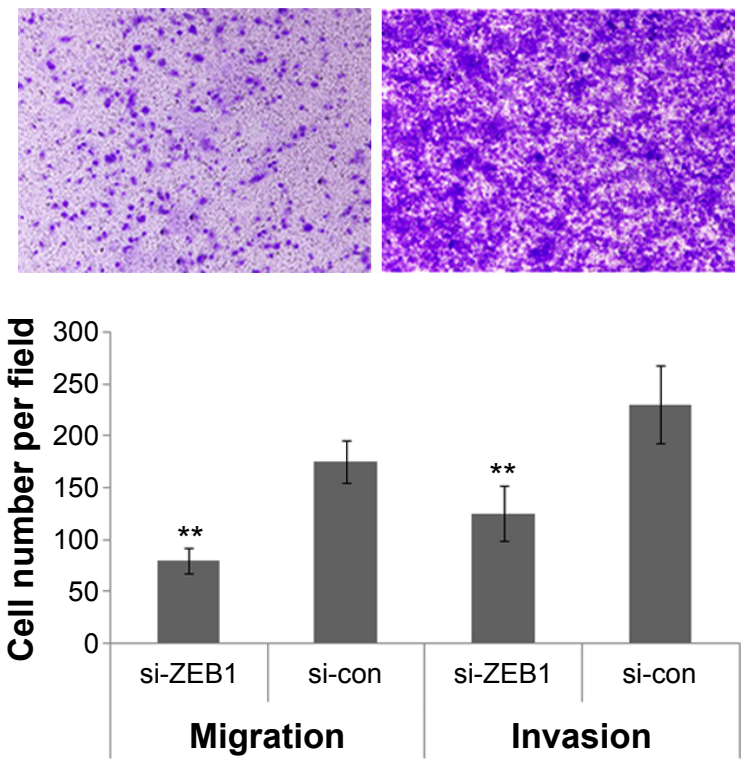

Figure 3 Knocking down ZEBI expression inhibits the migration and invasion of ARO (A) and TT (B) cells in vitro. Note: $* * P<0.01$ when compared with control cells.

Abbreviation: ZEBI, zinc finger E-box-binding homeobox I.

novel expression of ZEB1 in bladder tumors and showed a role for ZEB1 in enhanced migration and invasion potential in in vitro assays; Jia et $\mathrm{l}^{19}$ indicated that the overexpression of ZEB1 was related to the occurrence and development as well as invasion and metastasis of gastric carcinoma;
Liu et $\mathrm{al}^{20}$ found that ZEB1 was highly expressed in lung adenocarcinoma tissue and cell lines compared with adjacent noncancerous region and the human lung fibroblast cell HLF cells, and also validated that ZEB1-siRNA could inhibit lung adenocarcinoma cell growth in vitro and in vivo; Chen et $\mathrm{a}^{21}$
A

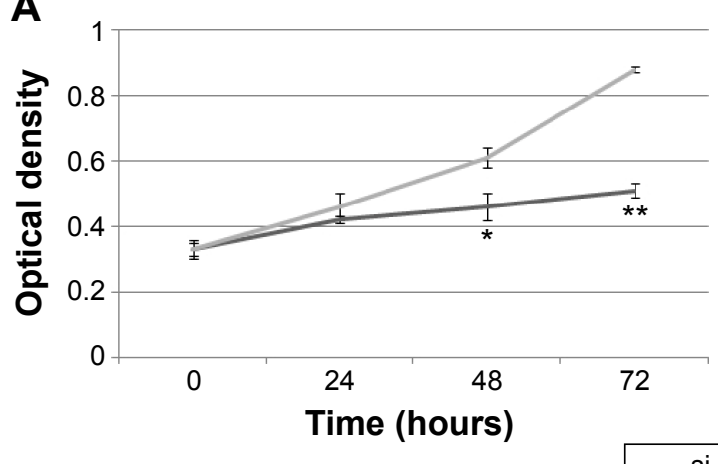

- si-ZEB1 si-con
B

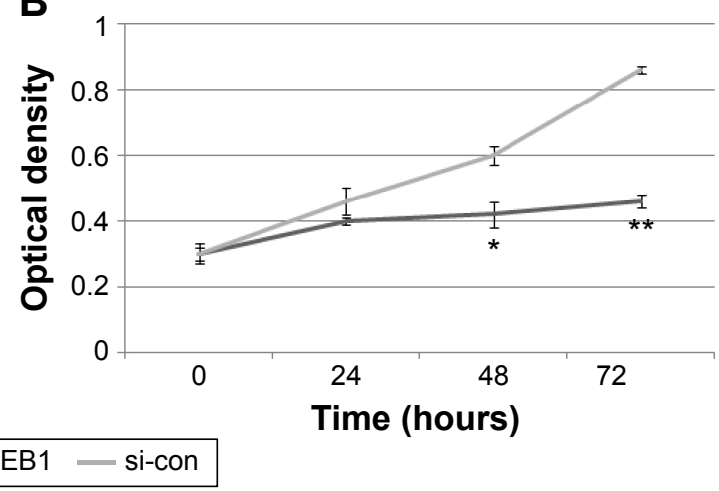

Figure 4 Knocking down ZEBI expression inhibits the proliferation of ARO (A) and TT (B) cells in vitro.

Notes: $* P<0.05$ and $* * P<0.01$ when compared with control cells.

Abbreviation: ZEBI, zinc finger E-box-binding homeobox I. 
proposed that ZEB1 had the potential to be used as a novel predictor of pelvic lymph node metastasis and represented promising therapeutic targets in patients with cervical cancer; Okugawa et $\mathrm{al}^{22}$ identified ZEB1 as an independent factor for peritoneal dissemination in patients with gastric cancer, and showed its roles in the progression to peritoneal dissemination in gastric cancer patients. In the current study, our immunohistochemistry analysis observed the positive immunostaining of ZEB1 localized in nucleus of cancer cells in human thyroid cancer tissues, which was in line with the previous findings in other cancer cells. ${ }^{16,18,19,22,23}$ Statistically, our data provides direct evidence that ZEB1 overexpression was significantly associated with the advanced TNM staging, the positive lymph node, and distant metastases. Since the prediction whether there is lymph node metastasis in the lateral compartment is of more clinical significance, it is necessary to take ZEB1 expression into consideration during surgery management decision.

Multiple factors and molecular events have been revealed to be implicated into cell migration and invasion, which are the most important biological characteristics of malignant tumors. ${ }^{24,25}$ Thus, to investigate the underlying molecular mechanisms of cell migration and invasion in thyroid cancer as well as to find new molecular therapeutic targets will improve treatment efficacy for this disease. Our above statistical analysis based on clinical samples showed the potential roles of ZEB1 overexpression in promoting aggressive tumor progression of thyroid cancer, which prompted us to further confirm whether ZEB1 could play any direct role in enhancing the migration and invasion abilities of thyroid cancer cells. Consistent with the previous findings on other cancer cells, ${ }^{18,19,26}$ we here confirmed the inhibitory effect of ZEB1 knockdown on thyroid cancer cell migration, invasion, and proliferation in vitro, although the underlying mechanisms and molecular events require further investigation.

\section{Conclusion}

Our findings indicated that ZEB1 might function as an oncogene, the overexpression of which was associated with the aggressive tumor progression of human thyroid cancer. Interestingly, ZEB1 also could promote thyroid cancer cell migration, invasion, and proliferation, suggesting that the inhibition of this protein might be a therapeutic strategy for the treatment of this malignancy.

\section{Disclosure}

The authors report no conflicts of interest in this work.

\section{References}

1. Boufraqech M, Patel D, Xiong Y, Kebebew E. Diagnosis of thyroid cancer: state of art. Expert Opin Med Diagn. 2013;7:331-342.

2. Kunavisarut T. Diagnostic biomarkers of differentiated thyroid cancer. Endocrine. 2013;44:616-622.

3. Ragazzi M, Ciarrocchi A, Sancisi V, Gandolfi G, Bisagni A, Piana S. Update on anaplastic thyroid carcinoma: morphological, molecular, and genetic features of the most aggressive thyroid cancer. Int J Endocrinol. 2014;2014:790834.

4. Nixon IJ, Ganly I, Shah JP. Thyroid cancer: surgery for the primary tumor. Oral Oncol. 2013;49:654658.

5. Jin J, Phitayakorn R, Wilhelm SM, McHenry CR. Advances in management of thyroid cancer. Curr Probl Surg. 2013;50:241-289.

6. Li L, Li W. Epithelial-mesenchymal transition in human cancer: comprehensive reprogramming of metabolism, epigenetics, and differentiation. Pharmacol Ther. 2015;150:33-46.

7. Schmalhofer O, Brabletz S, Brabletz T. E-cadherin, beta-catenin, and ZEB1 in malignant progression of cancer. Cancer Metastasis Rev. 2009; 28:151-166.

8. Wong TS, Gao W, Chan JY. Transcription regulation of E-cadherin by zinc finger E-box binding homeobox proteins in solid tumors. Biomed Res Int. 2014;2014:921564.

9. Cervantes-Arias A, Pang LY, Argyle DJ. Epithelial-mesenchymal transition as a fundamental mechanism underlying the cancer phenotype Vet Comp Oncol. 2013;11:169-184.

10. Guan H, Liang W, Xie Z, et al. Down-regulation of miR-144 promotes thyroid cancer cell invasion by targeting ZEB1 and ZEB2. Endocrine. 2015;48:566-574.

11. Meng X, Kong DH, Li N, et al. Knockdown of BAG3 induces epithelialmesenchymal transition in thyroid cancer cells through ZEB1 activation. Cell Death Dis. 2014;5:e1092.

12. Jiang SS, Weng DS, Wang QJ, et al. Galectin-3 is associated with a poor prognosis in primary hepatocellular carcinoma. J Transl Med. 2014; 12:273.

13. Remacle JE, Kraft H, Lerchner W, et al. New mode of DNA binding of multi-zinc finger transcription factors: deltaEF 1 family members bind with two hands to two target sites. EMBO J. 1999;18:5073-5084.

14. Postigo AA, Depp JL, Taylor JJ, Kroll KL. Regulation of Smad signaling through a differential recruitment of coactivators and corepressors by ZEB proteins. EMBO J. 2003;22:2453-2462.

15. Cao Z, Fu B, Deng B, Zeng Y, Wan X, Qu L. Overexpression of Chemokine (C-X-C) ligand 1 (CXCL1) associated with tumor progression and poor prognosis in hepatocellular carcinoma. Cancer Cell Int. 2014;14:86.

16. Liu Z, Sun B, Qi L, Li H, Gao J, Leng X. Zinc finger E-box binding homeobox 1 promotes vasculogenic mimicry in colorectal cancer through induction of epithelial-to-mesenchymal transition. Cancer Sci. 2012; 103:813-820.

17. Zhang W, Shi X, Peng Y, et al. HIF-1 $\alpha$ promotes epithelial-mesenchymal transition and metastasis through direct regulation of ZEB1 in colorectal cancer. PLoS One. 2015;10:e0129603.

18. Kenney PA, Wszolek MF, Rieger-Christ KM, et al. Novel ZEB1 expression in bladder tumorigenesis. BJU Int. 2011;107:656-663.

19. Jia B, Liu H, Kong Q, Li B. Overexpression of ZEB1 associated with metastasis and invasion in patients with gastric carcinoma. Mol Cell Biochem. 2012;366:223-229.

20. Liu Y, Yan X, Liu N, et al. Lentivirus-delivered ZEB-1 small interfering RNA inhibits lung adenocarcinoma cell growth in vitro and in vivo. J Cancer Res Clin Oncol. 2012;138:1329-1338.

21. Chen Z, Li S, Huang K, et al. The nuclear protein expression levels of SNAI1 and ZEB1 are involved in the progression and lymph node metastasis of cervical cancer via the epithelial-mesenchymal transition pathway. Hum Pathol. 2013;44:2097-2105.

22. Okugawa $Y$, Toiyama $Y$, Tanaka $K$, et al. Clinical significance of Zinc finger E-box binding homeobox 1 (ZEB1) in human gastric cancer J Surg Oncol. 2012;106:280-285. 
23. Quan Y, Jin R, Huang A, et al. Downregulation of GRHL2 inhibits the proliferation of colorectal cancer cells by targeting ZEB1. Cancer Biol Ther. 2014;15:878-887.

24. Han T, Kang D, Ji D, et al. How does cancer cell metabolism affect tumor migration and invasion? Cell Adh Migr. 2013;7:395-403.
25. Fife CM, McCarroll JA, Kavallaris M. Movers and shakers: cell cytoskeleton in cancer metastasis. Br J Pharmacol. 2014;171:5507-5523.

26. Paek AR, Lee $\mathrm{CH}$, You HJ. A role of zinc-finger protein 143 for cancer cell migration and invasion through ZEB1 and E-cadherin in colon cancer cells. Mol Carcinog. 2014;53(Suppl 1):E161-E168.

\section{Publish your work in this journal}

OncoTargets and Therapy is an international, peer-reviewed, open access journal focusing on the pathological basis of all cancers, potential targets for therapy and treatment protocols employed to improve the management of cancer patients. The journal also focuses on the impact of management programs and new therapeutic agents and protocols on

\section{Dovepress}

patient perspectives such as quality of life, adherence and satisfaction. The manuscript management system is completely online and includes a very quick and fair peer-review system, which is all easy to use. Visit http://www.dovepress.com/testimonials.php to read real quotes from published authors.

Submit your manuscript here: http://www.dovepress.com/oncotargets-and-therapy-journal 